\title{
Enlighting the transverse structure of the proton via double parton scattering in photon-induced interactions
}

\author{
F. A. Ceccopieri॰ \\ Université Paris-Saclay, CNRS, IJCLab, 91405 Orsay, France and IFPA, \\ Univèrsité de Liège, B4000 Liége, Belgium \\ M. Rinaldi $\odot^{*}$ \\ Dipartimento di Fisica e Geologia, Università degli studi di Perugia, \\ INFN section of Perugia, Via A. Pascoli, Perugia 06123, Italy
}

(Received 16 April 2021; accepted 14 December 2021; published 7 January 2022)

\begin{abstract}
In the present paper we address double parton scattering (DPS) in quasireal photon-proton interactions. By using electromagnetic and hadronic models of the photon light cone wave functions, we compute the so-called effective cross section, $\sigma_{\text {eff }}^{\gamma p}$ which allows us to calculate the DPS contribution to these processes under dedicated assumptions. In particular, for the four-jet photoproduction in HERA kinematics we found a sizeable DPS contribution. We show that if the photon virtuality $Q^{2}$ could be measured and thus the dependence of $\sigma_{\mathrm{eff}}^{\gamma p}$ on such a parameter exposed, information on the transverse distance between partons active in proton could be extracted. To this aim, we set lower limits on the integrated luminosity needed to observe such an effect which would allow the extraction of novel information on the proton structure.
\end{abstract}

DOI: 10.1103/PhysRevD.105.L011501

\section{INTRODUCTION}

There are increasing experimental evidences that multiple parton interactions (MPI) may occur within the same hadronic collision as a result of the composite and extended nature of the colliding hadrons. After preliminary investigations [1-6], the inclusion of MPI has been proven to be required to obtain a proper description of the multiplicity and topology of the hadronic final state of $p p$ collisions at collider energies $[7,8]$. Whereas MPI are characterized by soft and semihard components, in the present paper we will focus on double parton scattering (DPS), in which both scatterings involve a large momentum transfer of the order of few $\mathrm{GeV}$, so that short distance cross sections are perturbatively calculable. DPS could unveil parton correlations in the hadron structure not accessible in singleparton scattering (SPS). Such correlations are encoded in novel distributions, i.e., double parton distribution functions (dPDFs) which appear in the DPS cross section. The latter are interpreted as the number densities of a parton pair with a given transverse distance $b_{\perp}$ and carrying

\footnotetext{
* Corresponding author. matteo.rinaldi@pg.infn.it

Published by the American Physical Society under the terms of the Creative Commons Attribution 4.0 International license. Further distribution of this work must maintain attribution to the author(s) and the published article's title, journal citation, and DOI. Funded by SCOAP ${ }^{3}$.
}

longitudinal momentum fractions $\left(x_{1}, x_{2}\right)$ of the parent hadron [9-15]. Despite the ongoing theoretical efforts to investigate dPDFs [16-21], the structure of the DPS cross section and its factorization properties [22-28], rather limited knowledge has been accumulated so far and DPS measurements have provided information mainly on $\sigma_{\text {eff }}$ in $p p$ collisions [29] and recently in $p A$ collisions [30]. This dimensionful parameter controls the magnitude of DPS contribution under the simplifying assumptions of two uncorrelated hard scatterings and full factorization of dPDFs in terms of ordinary PDFs and model-dependent distribution in transverse position space. It has been shown in Refs. [31,32] that the knowledge of $\sigma_{\text {eff }}$ can provide information on the proton structure, complementary to that obtained from generalized parton distribution functions. For the final state relevant to this analysis, i.e., four-jet production in $p p$ collisions, recent experimental results are in the range $\sigma_{\text {eff }} \sim 8 \mathrm{mb}-35 \mathrm{mb}[33,34]$.

We propose here a strategy to extract novel information on the partonic structure of the proton by considering DPS processes in photon-proton interactions. In such a process, the impact of MPI has been studied in Ref. [35] via Monte Carlo simulations, whereas the DPS contribution has been considered in Ref. [36] in the direct photon kinematics, in which the DPS processes is initiated by a $c \bar{c}$ pair originating from the perturbative splitting of the photon. It is well known that in high-energy reactions a quasireal photon exhibits a rather complex hadronic structure [37]. It can interact as a pointlike particle with partons 
in the hadronic target, but it can also resolve into a hadronic structure and its partonic constituents could participate in the hard scattering. Additionally, as far as the (low) virtuality $Q^{2}$ of the photon can be measured by tagging the photon-emitter particles, the average transverse size of the $q \bar{q}$ pair fluctuation, $\left\langle b_{\perp}^{2}\right\rangle_{\gamma}$ can be controlled, since it scales as $1 / Q^{2}[38,39]$. Therefore, we address the intriguing question whether a DPS contribution could be observed in quasireal photon-proton interactions, in full analogy with hadronic ones. In the present paper we will consider $e p$ collisions where the electron is the photon emitter. The generalization to other reactions involving nucleon and/or nuclei only requires the use of the appropriate photon flux factors. In such a favorable environment the DPS mechanism, which is especially sensitive to parton pair correlations in transverse plane of the colliding particles, could be studied with a projectile of variable and controllable transverse size. Since a complete formulation of photon dPDFs capturing its longitudinal and transverse structure accommodating both its electromagnetic and hadronic components is missing at the moment, in the present paper we elaborate on a much simpler quantity, $\sigma_{\text {eff }}^{\gamma p}$.

With those results at hand, we present a first estimate of the DPS cross section for the photoproduction of four-jet in HERA kinematics accompanied by its main background, i.e., the SPS four-jet photoproduction cross-section, which, to the best of our knowledge, has never being discussed in the literature. A reliable estimate of the latter gives, in fact, a limit on the DPS contribution and thus constrains the corresponding models both of the photon and of the proton. We then show that if the $Q^{2}$ dependence of $\sigma_{\text {eff }}^{\gamma p}$ could be measured, then a first estimate of the mean transverse distance between partons in the proton could be obtained. Such a procedure avoids the intrinsic limitations in the extraction of this quantity from $\sigma_{\text {eff }}$ which are discussed in Refs. [31,32]. Moreover we derived lower limits on the necessary integrated luminosity to observe the predicted $Q^{2}$ dependence of the DPS cross sections in HERA kinematics.

\section{EFFECTIVE CROSS SECTION FOR $\gamma p$ DPS}

The $\gamma p$ DPS cross section, $\sigma_{\mathrm{DPS}}^{\gamma p}$, can be written in full analogy with the one appearing in the $p p$ case [7]. The former does depend on both the proton and photon dPDFs, $D_{q_{i} q_{j} / p}\left(x_{i}, x_{j}, k_{\perp}\right)$ and $D_{q \bar{q} / \gamma}\left(x_{k}, x_{l}, k_{\perp}\right)$, respectively where $i j$ and $k l$ are the flavors of the interacting partons, $k_{\perp}$ is the momentum imbalance Fourier conjugate variable to the partonic transverse distance, $b_{\perp}$ and $x$ are the longitudinal momentum fractions carried by each parton. The photon contribution to the cross section can be formally written similarly to that of meson dPDFs [40,41]. In particular, the light-front (LF) wave functions of the photon can be generally treated as that of a vector meson. With this respect, the dPDFs of the $\rho$ meson have been investigated in Ref. [42]. We leave for future analyses the study of the rich spin structure of these vector systems which could give access to new double-spin correlations in the proton.

The pair production amplitude, at a given photon virtuality $Q^{2}$, can be described within a light-front formalism in terms of the LF wave function, $\psi^{\gamma}$ [40],

$$
\begin{aligned}
D_{q \bar{q} / \gamma}\left(x, \vec{k}_{\perp} ; Q^{2}\right)= & \int d^{2} \vec{k}_{\perp, 1} \psi_{q \bar{q}}^{\dagger \gamma}\left(x, \vec{k}_{\perp, 1} ; Q^{2}\right) \\
& \times \psi_{q \bar{q}}^{\gamma}\left(x, \vec{k}_{\perp, 1}+\vec{k}_{\perp} ; Q^{2}\right) .
\end{aligned}
$$

In the above equation we take into account the lowest Fock components. This in turn implies that, being a twoparticle state, the longitudinal momentum of the second parton is given by $1-x$. The integration runs over the intrinsic transverse momentum of one parton of the pair, $\vec{k}_{\perp, 1}$, with $\vec{k}_{\perp, 2}=-\vec{k}_{\perp, 1}$.

Given the LF description of the unpolarized dPDFs and PDFs [19,40], one can derive the expression of the effective cross section in terms of effective form factors (effs) [43]. The latter, for the photon, reads

$$
F_{2}^{\gamma}\left(\vec{k}_{\perp}\right)=\frac{\sum_{q} \int d x D_{q \bar{q} / \gamma}\left(x, \vec{k}_{\perp}\right)}{\sum_{q} \int d x D_{q \bar{q} / \gamma}\left(x, \vec{k}_{\perp}=0\right)},
$$

in which the summation and integration, at variance with proton case, run over the indices of only one parton of the pair. Such a definition of the eff relies on the approximation, frequently assumed in phenomenological analyses in the $p p$ case, that momentum correlations and parton flavor dependence are neglected. Moreover, it guarantees that $F_{2}^{\gamma}\left(\vec{k}_{\perp}=0\right)=1$, as required by the probabilistic interpretation of double parton distributions in coordinate space and their corresponding normalization. In terms of these quantities, the $\gamma p$ effective cross sections can be written as

$$
\sigma_{\mathrm{eff}}^{\gamma p}\left(Q^{2}\right)=\left[\int \frac{d^{2} k_{\perp}}{(2 \pi)^{2}} F_{2}^{p}\left(k_{\perp}\right) F_{2}^{\gamma}\left(k_{\perp} ; Q^{2}\right)\right]^{-1} .
$$

Under the additional assumption that double PDFs can be written, at any perturbative scale, as product of ordinary PDFs, the $\gamma p$ DPS cross section for the production of the final state $A+B$ is rearranged in a pocket formula $\sigma_{\mathrm{DPS}}^{A+B} \sim \sigma_{\mathrm{SPS}}^{A} \sigma_{\mathrm{SPS}}^{B} / \sigma_{\mathrm{eff}}^{\gamma p}$. Such an approximation allows an estimation of $\sigma_{\text {DPS }}$ by making use of known calculations of SPS cross sections $\sigma_{\text {SPS }}^{A(B)}$ with $A(B)$ final states. It is worth remarking that such a procedure, largely used in DPS in $p p$ collisions and also adopted here, neglects any type of perturbative and nonperturbative correlations in double PDFs. 


\section{NUMERICAL RESULTS FOR $\sigma_{\mathrm{eff}}^{\gamma p}$}

The evaluation of $\sigma_{\mathrm{eff}}^{\gamma p}\left(Q^{2}\right)$ requires the knowledge of the proton effective form factor for which we use phenomenological parametrizations. In particular we consider the dipole one of Ref. [15], which we address as model "S"

$$
F_{2}^{p}\left(\vec{k}_{\perp}\right)=\left(1+k_{\perp}^{2} / m_{g}^{2}\right)^{-4},
$$

with $m_{g}^{2}=1.1 \mathrm{GeV}^{2}$. Such a model returns a $\sigma_{\mathrm{eff}}^{p p} \sim 30 \mathrm{mb}$. In addition, in order to explore the dependence of our results on the functional dependence of the proton eff, we also considered a Gaussian ansatz [29] of the type

$$
F_{2}^{p}\left(\vec{k}_{\perp}\right)=e^{-\alpha_{i} k_{\perp}^{2}}, \quad i=1,2 .
$$

The parameter $\alpha$ is fixed to $\alpha_{1}=1.53 \mathrm{GeV}^{-2}$ which returns $\sigma_{\text {eff }}^{p p}=15 \mathrm{mb}$ (" $\mathrm{G}_{1}$ " model) and to $\alpha_{2}=2.56 \mathrm{GeV}^{-2}$ which returns $\sigma_{\text {eff }}^{p p}=25 \mathrm{mb}$ ("G $\mathrm{G}_{2}$ " model). All considered proton effs satisfy the normalization condition $F_{2}^{p}\left(\vec{k}_{\perp}=0\right)=1$.

The other input appearing in Eq. (3) is the photon eff. The latter is calculated making use of the photon wave functions presented in Refs. [44,45]. Among those presented in Ref. [44], we make use of the wave functions corresponding to the so called "spectral-quark model". In Ref. [45] those quantities were evaluated to lowest-order QED in momentum space. As it is well known [39], the modulus squared of the corresponding wave function is logarithmically divergent at large parton transverse momentum $k_{\perp, 1}$ in Eq. (1). One option to regulate it is by introducing an upper physical cutoff on the $k_{\perp, 1}$ integration, possibly of the order of the hard scale entering the scattering process. In the present work we pursued instead the idea of considering a large $k_{\text {cut }}$ for the reason to be detailed hereafter. When increasingly higher cutoffs are used in the evaluation of $F_{2}^{\gamma}$, its tail at large $k_{\perp}$ shows, as expected, a sensitivity to $k_{\text {cut }}$ and it approaches a constant value of 1 for asymptotically large values of $k_{\text {cut }}$, i.e., the form factor of a structureless photon. However, the corresponding variations on the $\gamma p$ effective cross section evaluated via Eq. (3) are much reduced since the fast falling behavior of the proton eff at high $k_{\perp}[15,31,32]$ effectively regulates the tail of the photon eff in the convolution integral in Eq. (3) and grants its convergence. Although a residual dependence of $\sigma_{\text {eff }}^{\gamma p}$ on $k_{\text {cut }}$ is still present, we have numerically verified that the effective cross section varies no more than $\sim 1 \mathrm{mb}$ when $k_{\text {cut }}$ is raised from $k_{\text {cut }}=50 \mathrm{GeV}$ to $k_{\text {cut }}=10^{3} \mathrm{GeV}$, with the latter being used as default value in the following. See further details on this topic in the supplemental material [46]. Given these observations, the advantage of using a large cutoff is twofold: firstly one avoids introducing a prescription for setting a physical cutoff and the resulting arbitrariness; secondly one obtains a lower limit on the $\gamma p$-effective

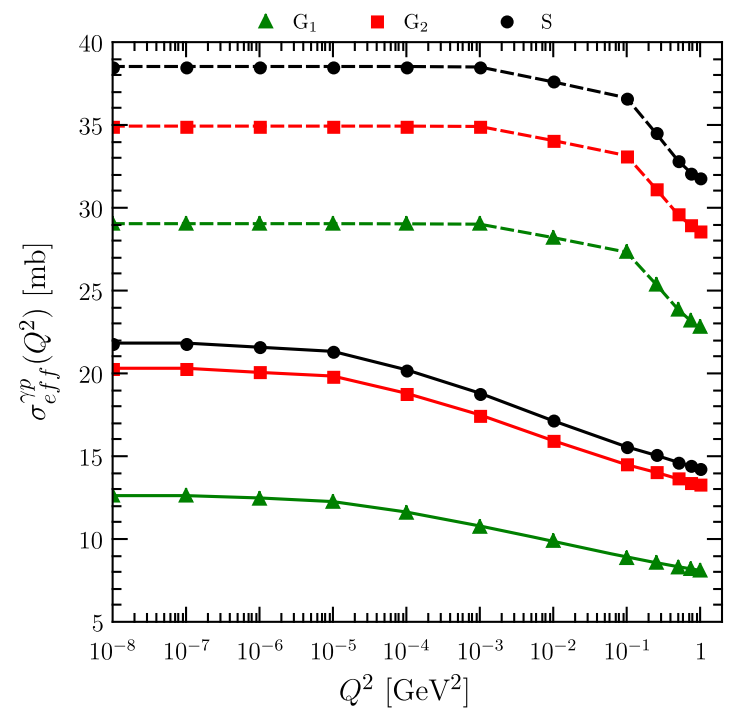

FIG. 1. $\sigma_{\text {eff }}^{\gamma p}$ evaluated in Eq. (3) with the wave function (w.f.) of Ref. [44] (dashed lines) and Ref. [45] (full lines) as a function of $Q^{2}$. Different symbols denote the proton effs described in the text.

cross section, which implies that our estimate of the DPS cross section given by QED contribution should be considered as its upper limit. This procedure is analogous to the one adopted in Ref. [39] in coordinate space, where the square modulus of the photon wave function, divergent in the small $b_{\perp}$ limit, is de facto regularized by the so-called dipole cross section which vanishes as $b_{\perp}$ goes to zero. In the case of hadronic model of the photon presented in Ref. [44] such an issue is not present, since the corresponding wave function is properly normalized from the beginning.

We present our numerical estimates for $\sigma_{\text {eff }}^{\gamma p}\left(Q^{2}\right)$ in Fig. 1. Since in the present paper we will consider photoproduction in ep collisions, the lower limit on $Q^{2}$ is set of the order of $m_{e}^{2}$, the mass of the electron, appearing in the Weizsäcker-Williams approximation for the spectrum of the exchanged photon. One may notice that the hadronic model of Ref. [44] returns a systematically higher $\sigma_{\mathrm{eff}}^{\gamma p}$ with respect to the pure electromagnetic one [45]. The spread between the curves pertinent to the same photon model indicates a rather large sensitivity to the proton effective form factor. Both models display a peculiar pattern of the $Q^{2}$ dependence; both start from a plateau at low $Q^{2}$ and decrease at larger $Q^{2}$, with the onset of the decrease occurring at rather different values of $Q^{2}$. The shape of the distribution is replicated irrespectively of the adopted proton eff. We observe that, in the limit of high photon virtuality, the value of $\sigma_{\text {eff }}^{\gamma p}$ can be predicted in complete analogy with the gluon splitting case elaborated in Ref. [47]. In fact, the $q \bar{q}$ pair, originated by the electromagnetic splitting of a highly virtual photon, is characterized by a quite small transverse distance, $\left\langle b_{\perp}^{2}\right\rangle_{\gamma} \propto 1 / Q^{2}$, and asymptotically one has $\left(\sigma_{\text {eff,asy }}\right)^{-1}=$ $\int d^{2} k_{\perp} /(2 \pi)^{2} F_{2}^{p}\left(k_{\perp}\right)=\tilde{F}_{2}^{p}\left(b_{\perp}=0\right)$ [47], where $\tilde{F}_{2}^{p}\left(b_{\perp}\right)$ 
is the Fourier transform of the eff and $b_{\perp}$ is the conjugate variable to $k_{\perp}$. Adopting the $\mathrm{G}_{1}$ model for the proton eff, the calculation with the photon wave function of Ref. [45] returns a value $\sigma_{\text {eff }}^{\gamma p}\left(Q^{2}=100 \mathrm{GeV}^{2}\right) \sim 7.52 \mathrm{mb}$ while the predictions from Ref. [47] would give $\sigma_{\text {eff,asy }} \sim 7.5 \mathrm{mb}$, showing remarkable agreement. We point out that the analysis of Ref. [36] is performed in the approximation described above, where the perturbative splitting of the photon into a $c \bar{c}$ pair probes the proton eff (model $S$ ) at zero transverse distance, which makes that analysis complementary to the one discussed here, where the quasireal photon develops a partonic structure at larger transverse distances.

\section{THE FOUR-JET PHOTO-PRODUCTION CROSS-SECTION}

The four-jet final states have been measured in photoproduction at HERA by the ZEUS Collaboration [48]. In that analysis, they considered jets with transverse energy $E_{T}^{\text {jet }}>6 \mathrm{GeV}$ and laboratory pseudorapidity $\left|\eta_{\text {jet }}\right|<2.4$, in the kinematic region $Q^{2}<1 \mathrm{GeV}^{2}$ and the energy fraction transferred from the lepton to the photon, $y=E_{\gamma} / E_{l}$, in the range $0.2 \leq y \leq 0.85$. The comparison with leadinglogarithmic parton-shower Monte Carlo models [35,49,50] showed that the inclusion in the simulation of multiparton interactions significantly improve the description of the data. This stimulated us to estimate the $\sigma_{\mathrm{DPS}}$ contribution to that final state by adopting kinematical cuts of Ref. [48]. The expression for the differential $\sigma_{\text {DPS }}$ initiated by a quasireal photon is then generalized according to the results presented in Ref. [47],

$$
\begin{aligned}
d \sigma_{\mathrm{DPS}}^{4 j}= & \frac{1}{2} \sum_{a b, c d} \int d y d Q^{2} \frac{f_{\gamma / e}\left(y, Q^{2}\right)}{\sigma_{\mathrm{eff}}^{\gamma p}\left(Q^{2}\right)} \\
& \times \int d x_{p_{a}} d x_{\gamma_{b}} f_{a / p}\left(x_{p_{a}}\right) f_{b / \gamma}\left(x_{\gamma_{b}}\right) d \hat{\sigma}_{a b}^{2 j}\left(x_{p_{a}}, x_{\gamma_{b}}\right) \\
& \times \int d x_{p_{c}} d x_{\gamma_{d}} f_{c / p}\left(x_{p_{c}}\right) f_{d / \gamma}\left(x_{\gamma_{d}}\right) d \hat{\sigma}_{c d}^{2 j}\left(x_{p_{c}}, x_{\gamma_{d}}\right) .
\end{aligned}
$$

In the above equation the one half factor takes into account two identical dijet systems in the final state. The sum runs over partons active in the first scattering $(a, b)$ or in the second one $(c, d)$, where $d \hat{\sigma}^{2 j}$ represent the differential partonic cross sections. Since $\sigma_{\text {eff }}^{\gamma p}$ depends on $Q^{2}$, the photon flux, $f_{\gamma / e}$, in its $Q^{2}$-unintegrated version [51] has been used. The distributions $f_{i / p}\left(x_{p_{i}}\right)$ and $f_{j / \gamma}\left(x_{\gamma_{j}}\right)$ represent the proton and of the photon PDFs for which we use the leading-order (LO) sets of Ref. [52,53], respectively. Dijet cross sections have been evaluated to leading-order accuracy in the strong coupling with ALPGEN [54], properly adapted to cope with photoproduction processes, with final state partons identified as jets. Factorization and
TABLE I. Predictions for the LO DPS and SPS cross sections for four-jet photo-production in three ranges of $Q^{2}$. In the last column, the ratio between the calculated cross sections to the total one is displayed. In the DPS case, each row corresponds to prediction obtained with a given $p p$ eff $\left(G_{1}, G_{2}, S\right)$, and the photon wave function of Refs. [44] (three upper rows) and Ref. [45] (three bottom rows). In the last column the ratio Eq. (7) is shown.

\begin{tabular}{lcccccc}
\hline \hline & & $\begin{array}{c}Q^{2} \leq 10^{-2} \\
{\left[\mathrm{GeV}^{2}\right]}\end{array}$ & $\begin{array}{c}10^{-2} \leq Q^{2} \leq 1 \\
{\left[\mathrm{GeV}^{2}\right]}\end{array}$ & $\begin{array}{c}Q^{2} \leq 1 \\
{\left[\mathrm{GeV}^{2}\right]}\end{array}$ & $\frac{\sigma}{\sigma_{\text {exp }}}[\%]$ & $R$ \\
\hline & & \multicolumn{7}{c}{$\sigma_{\text {DPS }}[\mathrm{pb}]$} \\
& $\mathrm{G}_{1}$ & 35.1 & 18.6 & 53.7 & 40 & 1.89 \\
w.f. & $\mathrm{G}_{2}$ & 29.1 & 15.2 & 44.3 & 33 & 1.91 \\
{$[44]$} & $\mathrm{S}$ & 26.4 & 13.7 & 40.1 & 30 & 1.93 \\
& & & & & & \\
& $\mathrm{G}_{1}$ & 87.8 & 54.3 & 142.1 & 101 & 1.62 \\
w.f. & $\mathrm{G}_{2}$ & 54.3 & 33.4 & 87.7 & 65 & 1.63 \\
{$[45]$} & $\mathrm{S}$ & 50.5 & 31.1 & 81.6 & 60 & 1.62 \\
& & \multicolumn{7}{c}{$\sigma_{\text {SPS }}[\mathrm{pb}]$} \\
LO SPS & 77.5 & 36.6 & 114.1 & 86 & 2.12 \\
\hline \hline
\end{tabular}

renormalization scales have been both set to average transverse momentum of the jets. Such a cross section receives two contributions that can be classified by the fractional momentum of partons in the photon, $x_{\gamma}$, reconstructed by jet kinematics; the one from the resolved photon process, in which the photon behaves like an hadron with its own parton distributions, and the direct one in which the photon interacts as a pointlike particle with partons in the proton target. The former populates the whole $x_{\gamma}$ range while the latter, at LO, is concentrated at $x_{\gamma}=1$. The two mechanisms mix under higher-order corrections [55] and therefore kinematic cuts are used by experimental collaborations [56,57] to select the resolved-enriched contribution $\left(x_{\gamma}<0.75\right)$ and a direct-enriched one $\left(x_{\gamma}>0.75\right)$. In the present analysis we are interested in the resolved component and therefore the cut $x_{\gamma}<0.75$ is enforced on the evaluation of dijet cross sections. Out of this prediction, the DPS cross section is built via the pocket formula in Eq. (6) by enforcing, for consistency, the same cut on the parton pair fractional momenta, $x_{\gamma, 1}+x_{\gamma, 2}<0.75$. The main background to the DPS signal is represented by the SPS four-jet photoproduction process. The latter is again calculated with ALPGEN interfaced with the photon flux factor and photon PDFs, enforcing $x_{\gamma}<0.75$ and with same settings discussed for the dijet cross sections. The experimental four-jet photoproduction cross section, $\left(\sigma_{\text {exp }}\right)$, that can be inferred from distributions presented in Ref. [48] for $x_{\gamma}<0.75$ is $135 \mathrm{pb}$.

We report in Table I the results for the $\sigma_{\mathrm{DPS}}$ and $\sigma_{\mathrm{SPS}}$ obtained for three ranges of photon virtualities in HERA kinematics. Predictions are displayed on different rows depending on the adopted proton eff and photon wave 
functions. As far as the comparison with the experimental cross section for $Q^{2}<1 \mathrm{GeV}^{2}$ is concerned, the DPS cross section gives a sizeable contribution for all configurations whereas the LO SPS almost saturates the experimental cross section. With this respect, this preliminary investigation already indicates that some configurations, e.g., the LO QED description of the photon combined with the $G_{1}$ proton eff, are not favorable combinations since the corresponding DPS cross section alone exceeds the experimental one.

These results, however, should be interpreted with special care. Higher-order corrections to the dijet photoproduction cross section induce an increase of theoretical predictions by a factor of 1.3 going from LO to next-to-leading order (NLO) $[58,59]$ and by a factor 1.05 going from NLO to nextto-next-to-Leading order (NNLO) [60]. This in turn implies that by using LO estimates for the dijet cross section in the pocket formula, the latter gives a lower limit on the DPS cross section, as far as higher-order corrections are considered. On the other hand, a good theoretical control of higherorder corrections to the SPS background is mandatory for a proper extraction of DPS signal. For example, the large spread in $\sigma_{\text {eff }}$ values reported in the experimental analysis of Ref. [34] reflects the level of uncertainty in the theoretical estimation of the $\sigma_{\mathrm{SPS}}^{4 j}$. With this respect, NLO results for four-jet production in $p p$ collisions at $8 \mathrm{TeV}$ were first calculated in Ref. [61,62] showing that NLO predictions are nearly half of the LO estimates. If such a trend should be confirmed also in four-jet photoproduction in HERA kinematics, our LO result would represent an upper limit on the SPS background. The scenario concerning the uncertainties connected to higher-order corrections appears as follows. Our LO estimates sets a lower limit on the DPS cross section, whereas we have presented arguments showing that the LO SPS could represent its upper bound. Both these findings converge into a conservative scenario for the DPS contribution to four-jet cross section. For those reasons we do not provide here a full propagation of theoretical uncertainties to our final results. Nevertheless, it is worth mentioning that the largest theoretical uncertainty, and by far dominant over all others, comes from a) models of the proton structure and the spread in the corresponding $\sigma_{\text {eff }}^{p p}$ values and b) the use of a LO QED treatment of the photon in addition to quark models for its hadronic component, without their consistent combination into a photon double PDFs, which in turns generates a wide spread on $\sigma_{\text {eff }}^{\gamma p}$ predictions.

Despite all these warnings, all models predict large DPS fractions suggesting that jet photoproduction in $e p$ collisions could represent an interesting channel to search for the DPS contribution.

\section{EXTRACTION OF THE $Q^{2}$-DEPENDENCE OF $\sigma_{\text {eff }}^{\gamma p}$}

Given the rather large uncertainties on the absolute DPS cross sections, it seems to us premature to consider more differential observable at this point. We make only one exception by discussing the $Q^{2}$-dependence of the DPS cross section. The latter is of primary importance to us since it is linked to the concept of a photon of variable transverse size and, in turn, to a $Q^{2}$-dependent $\sigma_{\text {eff }}^{\gamma p}$. We investigate whether such a dependence, which adds on top of the one naturally induced by the photon flux, is eventually observable. We perform such an analysis within the HERA settings presented in the previous section. We set the notation by sketching Eq. (6) as $d \sigma_{\text {DPS }}($ bin $) \sim \int_{\text {bin }} d Q^{2} g\left(Q^{2}\right) / \sigma_{\text {eff }}^{\gamma p}\left(Q^{2}\right)$, where bin stands for a given interval of integration over $Q^{2}$ and the function $g$ encodes the flux factor, the PDFs, and elementary cross sections. We present in the first two columns of Table I the DPS cross section integrated over ranges of photon virtualities. Then we define the ratio $R$,

$$
R=\frac{d \sigma_{\mathrm{DPS}}(\operatorname{bin} 1)}{d \sigma_{\mathrm{DPS}}(\operatorname{bin} 2)}
$$

In the case $\sigma_{\text {eff }}^{\gamma p}$ were a constant, the latter quantity would be: $R \sim \int_{\text {bin } 1} d Q^{2} g\left(Q^{2}\right) / \int_{\text {bin2 }} d Q^{2} g\left(Q^{2}\right) \sim 2.1$. Therefore, if the ratio of the DPS cross sections, evaluated in the two bins, results to be different from that number, this fact would directly point to $Q^{2}$ effects on $\sigma_{\text {eff }}^{\gamma p}$ or possible correlations breaking the pocket formula. As one can see in the last column of Table I, LO QED and the modelencoding nonperturbative QCD effects predict deviations of $R$ from the reference value of 2.1. We close this section remarking that this ratio is particular effective since it does not depend on the chosen proton and photon effs-it is sensitive to the dependence of $\sigma_{\text {eff }}^{\gamma p}$ on $Q^{2}$ and finally, if applied to the SPS four-jet cross section, its value gives a theoretical benchmark without requiring the exact knowledge of the absolute four-jet cross section.

Furthermore, we also developed a procedure to establish the minimum integrated luminosity to experimentally access $Q^{2}$ effects in $\sigma_{\text {eff }}^{\gamma p}\left(Q^{2}\right)$. We have converted the cross sections in Table I in the expected number of events with a given integrated luminosity. Statistical errors and the corresponding bands are calculated assuming a Poisson distribution. The results are presented in Fig. 2 where the blue curves indicate results for the $\sigma_{\text {eff }}^{\gamma p}\left(Q^{2}\right)$ while red ones indicate the number of events obtained with a constant $Q^{2}$ independent $\bar{\sigma}_{\text {eff }}^{\gamma p}$ which reproduces the total cross section for $Q^{2}<1 \mathrm{GeV}^{2}$ obtained with $\sigma_{\text {eff }}^{\gamma p}\left(Q^{2}\right)$. Among the various model results listed in Table I, in Fig. 2 it is shown, on purpose, the one with the lower cross section and with a smooth $Q^{2}$ dependence. We find that the minimal integrated luminosity which makes distinguishable the two models giving nonoverlapping error bands, and therefore exposes the $Q^{2}$-dependence of $\sigma_{\text {eff }}^{\gamma p}$, is $\mathcal{L}=200 \mathrm{pb}^{-1}$.

This integrated luminosity estimate assumes that the SPS background could be subtracted with high efficiency and 


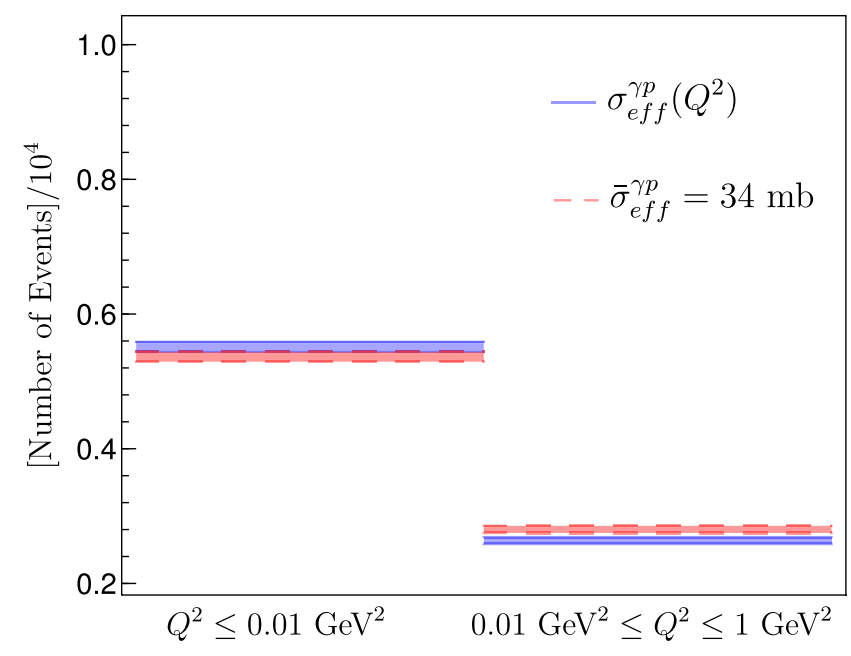

FIG. 2. The estimated number of events as a function of $Q^{2}$ for $200 \mathrm{pb}^{-1}$ of integrated luminosity for the photon model of Ref. [44] and proton eff G2. Solid lines represent the evaluations of $\sigma_{\text {DPS }}$ by means of $\sigma_{\mathrm{eff}}^{\gamma p}\left(Q^{2}\right)$ and the dotted ones represent the calculations of $\sigma_{\text {DPS }}$ by using the $Q^{2}$-independent $\bar{\sigma}_{\text {eff }}^{\gamma p}$.

therefore it should be considered as a lower limit to start observing $Q^{2}$ effects in $\sigma_{\text {eff }}$.

\section{THE GEOMETRY OF $\sigma_{\text {eff }}^{\gamma p}\left(Q^{2}\right)$}

As discussed in Refs. [31,32], the rather limited knowledge of the proton eff entering the definition of $\sigma_{\text {eff }}^{p p}$ prevents a precise extraction of $\left\langle b_{\perp}^{2}\right\rangle_{p}$. In fact, in $p p$ or $p A$ collisions, the integrand defining the relative $\sigma_{\text {eff }}$, see e.g., Eq. (3), is completely unknown. Therefore, without assuming some model constraints, one cannot directly relate $\left\langle b_{\perp}^{2}\right\rangle_{p}$ to experimental data. The possibility to have in $\gamma p$ interactions a projectile of variable size, depending on $Q^{2}$, can provide a unique chance to extract $\left\langle b_{\perp}^{2}\right\rangle_{p}$. Without specifying any peculiar photon w.f., one may define the Fourier transform of the eff, $\tilde{F}_{2}\left(b_{\perp}\right)$, which is interpreted as the probability distribution of finding two partons at a given transverse distance $b_{\perp}[29,31,32]$. Once this quantity has been evaluated within some model of the photon structure, it can be power expanded as

$$
\tilde{F}_{2}^{\gamma}\left(b_{\perp} ; Q^{2}\right)=\sum_{n} C_{n}\left(\bar{b}_{\perp} ; Q^{2}\right)\left(b_{\perp}-\bar{b}_{\perp}\right)^{n},
$$

and freedom is left in the choice in the expansion point $\bar{b}_{\perp}$. Equation (3) can now be rewritten as

$$
\begin{aligned}
{\left[\sigma_{\mathrm{eff}}^{\gamma p}\left(Q^{2}\right)\right]^{-1} } & =\int d^{2} b_{\perp} \tilde{F}_{2}^{p}\left(b_{\perp}\right) \tilde{F}_{2}^{\gamma}\left(b_{\perp} ; Q^{2}\right) \\
& =\sum_{n} C_{n}\left(\bar{b}_{\perp} ; Q^{2}\right)\left\langle\left(b_{\perp}-\bar{b}_{\perp}\right)^{n}\right\rangle_{p}
\end{aligned}
$$

A realistic description of $C_{n}\left(\bar{b}_{\perp} ; Q^{2}\right)$, together with data on the $Q^{2}$ dependence of $\sigma_{\text {eff }}^{\gamma p}\left(Q^{2}\right)$, will allow us to access the transverse distance of partons in the proton. In fact, for a given specific dependence of $C_{n}$ on $Q^{2}$ one can identify an operator, $\mathcal{O}_{Q^{2}}^{m}$, such that

$\mathcal{O}_{Q^{2}}^{m}\left[\sigma_{\text {eff }}^{\gamma p}\left(Q^{2}\right)\right]^{-1}=\mathcal{O}_{Q^{2}}^{m} C_{m}\left(\bar{b}_{\perp}, Q^{2}\right)\left\langle\left(b_{\perp}-\bar{b}_{\perp}\right)^{m}\right\rangle_{p}$,

and then one can select and extract $\left\langle\left(b_{\perp}-\tilde{b}_{\perp}\right)^{m}\right\rangle_{p}$, i.e., the relevant information on the proton structure. For the moment being we do not specify any functional expression of $\mathcal{O}_{Q^{2}}^{m}$. This quantity, related to the explicit expressions of $C_{m}\left(\bar{b}_{\perp}, Q^{2}\right)$, could be e.g., a proper differential operator on $Q^{2}$. We have successfully tested the procedure both analytically and numerically with different proton and photon eff models. The identification of the correct operator is however not unique and freedom is left in the choice of the expansion point. Such a flexible feature can be useful for possible experimental applications. The only practical limitation is represented by the accuracy with which the dependence of $\sigma_{\text {eff }}^{\gamma p}$ on $Q^{2}$ could be eventually measured. Therefore, this procedure should be properly optimized along the experimental extraction conditions. In closing this section, we stress again that the procedure can be used only by considering realistic description of the photon-splitting mechanism by taking into account higherorder QED effects. Examples of application of this procedure are discussed in the supplemental material [46].

This relation is one of the main goals of the present analysis and constitutes motivation to suggest this type of measurements at facilities where the photon virtuality can be experimentally measured such as the future Electron Ion Collider [63].

\section{SUMMARY}

In the present analysis we have derived effective cross sections for photon-induced processes which are essential ingredients in the predictions of DPS cross sections in quasireal photon proton interactions. The latter has been obtained with the help of electromagnetic and hadronic model of the photon formulated in terms of light cone wave functions. For the four-jet final state in HERA kinematics we found a sizeable DPS contribution. This conclusion persists after considering estimates of higher-order corrections, both to the DPS and SPS processes, taken from the literature. In the case where the photon virtuality $Q^{2}$ could be measured, we have investigated the dependence of $\sigma_{\text {eff }}^{\gamma p}$ on such a parameter, which is directly related to the size of the dipole originated by the photon fluctuation. We set lower limits on the integrated luminosity needed to observe such an effect and we present, for such a case, a novel procedure which would allow to extract new information on the proton structure. 


\section{ACKNOWLEDGMENTS}

This work was supported: $i$ ) in part by the STRONG2020 project of the European Union Horizon 2020 research and innovation programme under Grant Agreement No. 824093; ii) by the European Research Council under the European Union's Horizon 2020 research and innovation program (Grant Agreement No. 804480); iii) by the project "Photon initiated double parton scattering: illuminating the proton parton structure" on the FRB of the University of Perugia.
[1] C. Goebel, F. Halzen, and D. M. Scott, Phys. Rev. D 22 , 2789 (1980).

[2] B. Humpert, Phys. Lett. 131B, 461 (1983).

[3] M. Mekhfi, Phys. Rev. D 32, 2371 (1985).

[4] M. Mekhfi, Phys. Rev. D 32, 2380 (1985).

[5] B. Humpert and R. Odorico, Phys. Lett. 154B, 211 (1985).

[6] M. L. Mangano, Z. Phys. C 42, 331 (1989).

[7] N. Paver and D. Treleani, Nuovo Cimento A 70, 215 (1982).

[8] T. Sjostrand and M. van Zijl, Phys. Lett. B 188, 149 (1987).

[9] M. Diehl, D. Ostermeier, and A. Schafer, J. High Energy Phys. 03 (2012) 089; 03 (2016) 001.

[10] M. Diehl and A. Schafer, Phys. Lett. B 698, 389 (2011).

[11] A. V. Manohar and W. J. Waalewijn, Phys. Rev. D 85, 114009 (2012).

[12] M. Rinaldi, S. Scopetta, and V. Vento, Phys. Rev. D 87, 114021 (2013).

[13] B. Blok, Yu. Dokshitser, L. Frankfurt, and M. Strikman, Eur. Phys. J. C 72, 1963 (2012).

[14] B. Blok, Yu. Dokshitzer, L. Frankfurt, and M. Strikman, Phys. Rev. D 83, 071501 (2011).

[15] B. Blok, Yu. Dokshitzer, L. Frankfurt, and M. Strikman, Eur. Phys. J. C 74, 2926 (2014).

[16] J. R. Gaunt and W. J. Stirling, J. High Energy Phys. 03 (2010) 005.

[17] H.-M. Chang, A. V. Manohar, and W. J. Waalewijn, Phys. Rev. D 87, 034009 (2013).

[18] M. Rinaldi, S. Scopetta, M. C. Traini, and V. Vento, J. High Energy Phys. 10 (2016) 063.

[19] M. Rinaldi, S. Scopetta, M. Traini, and V. Vento, J. High Energy Phys. 12 (2014) 028.

[20] M. Diehl, J. R. Gaunt, D. M. Lang, P. Plöß1, and A. Schäfer, Eur. Phys. J. C 80, 468 (2020).

[21] M. Diehl, P. Plößl, and A. Schäfer, Eur. Phys. J. C 79, 253 (2019).

[22] M. Diehl, J. R. Gaunt, and K. Schönwald, J. High Energy Phys. 06 (2017) 083.

[23] M. Diehl, J. R. Gaunt, D. Ostermeier, P. Plößl, and A. Schäfer, J. High Energy Phys. 01 (2016) 076.

[24] J. R. Gaunt, J. High Energy Phys. 07 (2014) 110.

[25] M. Diehl, J. R. Gaunt, P. Plöß1, and A. Schäfer, SciPost Phys. 7, 017 (2019).

[26] M. Diehl and R. Nagar, J. High Energy Phys. 04 (2019) 124.

[27] M. G. Ryskin and A. M. Snigirev, Phys. Rev. D 83, 114047 (2011).

[28] M. G. Ryskin and A. M. Snigirev, Phys. Rev. D 86, 014018 (2012).

[29] G. Calucci and D. Treleani, Phys. Rev. D 60, 054023 (1999).
[30] R. Aaij et al. (LHCb Collaboration), Phys. Rev. Lett. 125, 212001 (2020).

[31] M. Rinaldi and F. A. Ceccopieri, Phys. Rev. D 97, 071501 (2018).

[32] M. Rinaldi and F. A. Ceccopieri, J. High Energy Phys. 09 (2019) 097.

[33] M. Aaboud et al. (ATLAS Collaboratin), J. High Energy Phys. 11 (2016) 110.

[34] CMS Collaboration, Report No. CMS-PAS-SMP-20-007, 2021.

[35] J. M. Butterworth, J. R. Forshaw, and M. H. Seymour, Z. Phys. C 72, 637 (1996).

[36] B. Blok and M. Strikman, Eur. Phys. J. C 74, 3214 (2014).

[37] M. Klasen, Rev. Mod. Phys. 74, 1221 (2002).

[38] L. L. Frankfurt and M. I. Strikman, Phys. Rep. 76, 215 (1981).

[39] N. N. Nikolaev and B. G. Zakharov, Z. Phys. C 49, 607 (1991).

[40] M. Rinaldi, S. Scopetta, M. Traini, and V. Vento, Eur. Phys. J. C 78, 781 (2018).

[41] T. Kasemets and A. Mukherjee, Phys. Rev. D 94, 074029 (2016).

[42] M. Rinaldi, Eur. Phys. J. C 80, 678 (2020).

[43] M. Rinaldi, S. Scopetta, M. Traini, and V. Vento, Phys. Lett. B 752, 40 (2016).

[44] A. E. Dorokhov, W. Broniowski, and E. Ruiz Arriola, Phys. Rev. D 74, 054023 (2006).

[45] S. J. Brodsky, L. Frankfurt, J. F. Gunion, A. H. Mueller, and M. Strikman, Phys. Rev. D 50, 3134 (1994).

[46] See Supplemental Material at http://link.aps.org/ supplemental/10.1103/PhysRevD.105.L011501 we provide instructive examples of the application of the procedure presented in Sec. VI and a detailed analysis on the dependence of the results of Sec. IV on the cut-offs for LO QED photon wave function.

[47] J. R. Gaunt, J. High Energy Phys. 01 (2013) 042.

[48] S. Chekanov et al. (ZEUS Collaboration), Nucl. Phys. B792, 1 (2008).

[49] G. Marchesini, B. R. Webber, G. Abbiendi, I. G. Knowles, M. H. Seymour, and L. Stanco, Comput. Phys. Commun. 67, 465 (1992).

[50] T. Sjostrand, P. Eden, C. Friberg, L. Lonnblad, G. Miu, S. Mrenna, and E. Norrbin, Comput. Phys. Commun. 135, 238 (2001).

[51] S. Frixione, M. L. Mangano, P. Nason, and G. Ridolfi, Phys. Lett. B 319, 339 (1993).

[52] J. Pumplin, D. R. Stump, J. Huston, H. L. Lai, P. M. Nadolsky, and W. K. Tung, J. High Energy Phys. 07 (2002) 012. 
[53] M. Gluck, E. Reya, and A. Vogt, Phys. Rev. D 46, 1973 (1992).

[54] M. L. Mangano, M. Moretti, F. Piccinini, R. Pittau, and A. D. Polosa, J. High Energy Phys. 07 (2003) 001.

[55] S. Frixione and G. Ridolfi, Nucl. Phys. B507, 315 (1997).

[56] S. Chekanov et al. (ZEUS Collaboration), Phys. Rev. D 76, 072011 (2007).

[57] A. Aktas et al. (H1 Collaboration), Phys. Lett. B 639, 21 (2006).

[58] M. Klasen and G. Kramer, Z. Phys. C 76, 67 (1997).
[59] M. Klasen, T. Kleinwort, and G. Kramer, Eur. Phys. J. Direct 1, 1 (1998).

[60] M. Klasen, G. Kramer, and M. Michael, Phys. Rev. D 89, 074032 (2014).

[61] S. Badger, B. Biedermann, P. Uwer, and V. Yundin, Phys. Lett. B 718, 965 (2013).

[62] Z. Bern, G. Diana, L. J. Dixon, F. Febres Cordero, S. Hoeche, D. A. Kosower, H. Ita, D. Maitre, and K. Ozeren, Phys. Rev. Lett. 109, 042001 (2012).

[63] R. Abdul Khalek et al., arXiv:2103.05419. 